\title{
On optical-absorption peaks in a nonhomogeneous thin-film solar cell with a two-dimensional periodically corrugated metallic backreflector
}

Faiz Ahmad, ${ }^{a}$ Tom H. Anderson, ${ }^{b}$ Benjamin J. Civiletti, ${ }^{b}$ Peter B. Monk, ${ }^{b}$ and Akhlesh Lakhtakia ${ }^{a, *}$ ${ }^{a}$ Pennsylvania State University, Department of Engineering Science and Mechanics, NanoMM-Nanoengineered Metamaterials Group, University Park, PA 16802, USA

${ }^{b}$ University of Delaware, Department of Mathematical Sciences, 501 Ewing Hall, Newark, DE 19716, USA

*akhlesh@psu.edu

\begin{abstract}
The rigorous coupled wave approach (RCWA) was implemented to investigate optical absorption in a triple- $p$ - $i$ - $n$-junction amorphous-silicon solar cell with a $2 \mathrm{D}$ metallic periodically corrugated backreflector (PCBR). Both total and useful absorptances were computed against the free-space wavelength $\lambda_{0}$ for both $s$ - and $p$-polarized polarization states. The useful absorptance in each of the three $p-i$ - $n$ junctions was also computed for normal as well as oblique incidence. Furthermore, two canonical boundary-value problems were solved for the prediction of guided-wave modes (GWMs): surface-plasmon-polariton waves and waveguide modes. Use of the doubly periodic PCBR enhanced both useful and total absorptances in comparison to a planar backreflector. The predicted GWMs were correlated with the peaks of the total and useful absorptances. The excitation of GWMs was mostly confined to $\lambda_{0}<700 \mathrm{~nm}$ and enhanced absorption. As excitation of certain GWMs could be correlated with the total absorptance but not with the useful absorptance, the useful absorptance should be studied while devising light-trapping strategies.
\end{abstract}

\section{Introduction}

Amorphous silicon (a-Si) thin-film solar cells provide a viable option to the $1^{\text {st }}$-generation crystalline-silicon (c-Si) solar cells [1], due to their ease of manufacturing and low cost. But the typical efficiency of a-Si thin-film solar cells is not as high as of c-Si solar cells, due to the high electron-hole recombination rate and low chargecarrier diffusion lengths in a-Si $[2,3]$. Consequently, light-trapping techniques are necessary to enhance the efficiency of a-Si thin-film solar cells. Several light-trapping strategies have been studied both experimentally and theoretically $[4,5]$. Anti-reflection coatings [6, 7, 8], textured front faces [9, 10], metallic periodically corrugated backreflectors (PCBRs) [11, 12, 13], particle plasmonics[14], surface plasmonics [15, 16, 17] and multiplasmonics $[18,19,20]$, and waveguide-mode excitation $[21,22,23]$ are attractive for trapping light in solar cells.

Of particular interest is the enhancement of the optical electric field through the excitation of two types of guided-wave modes (GWMs): surface-plasmon-polariton (SPP) waves and waveguide modes (WGMs). The periodically corrugated interface of a metal and a semiconductor that is periodically nonhomogeneous in the thickness direction (identified by the $z$ axis in Sec. 2) can guide multiple SPP waves at the same frequency[18, 24]. Any open-face waveguide with an air/semiconductor/metal architecture can guide WGMs [22, 23, 25]. Therefore, the incorporation of nonhomogeneity along the thickness direction in the semiconductor layers of a solar cell with a PCBR can enhance photonic absorption $[18,21,26]$. That enhancement would increase the generation rate of electron-hole pairs [27, 26].

Much of the theoretical and experimental research done on thin-film solar cells with metallic PCBRs is confined to devices with a homogeneous semiconductor layer and a metallic backreflector with onedimensionally (1D) periodic corrugation. An experimental report of broadband excitation of multiple SPP waves in a device comprising a 1D photonic crystal (PC) atop a 1D PCBR [28] confirmed theoretical predictions [29] and spurred research on solar cells containing piecewise nonhomogeneous semiconductor layers and 1D PCBRs $[18,26,27,30]$. In a recent study, experimental excitation of multiple SPP waves and WGMs were reported in a device comprising a 1D PC atop a 2D PCBR [21]. Appropriately designed 2D PCBRs were found to be better for the excitation of GWMs than 1D PCBRs, after the broadband excitation of GWMs predicted by solving two canonical boundary-value problems was correlated with the experimentally measured absorption spectrums. 
In solar-cell research, often the excitation of GWMs is correlated with the total absorptance $\bar{A}^{\text {tot }}$ of the device $[18,23]$, which however is not a good measure of useful photonic absorption in a solar cell, as photons absorbed in the metallic portions of a solar cell are not available for conversion into electric current. Therefore, the chief objective for the work reported in this paper was to determine the spectrums of both the total absorptance $\bar{A}^{\text {tot }}$ and the useful absorptance $\bar{A}^{\text {sc }}[31]$ in a tandem solar cell with a $2 \mathrm{D}$ PCBR exposed to either normally or obliquely incident linearly polarized light. The solar cell was taken to comprise three $p$ - $i$ $n$ solar cells made of a-Si alloys [32] that can be fabricated using plasma-enhanced chemical-vapor deposition over planar and patterned substrates. A top layer of aluminum-doped zinc oxide (AZO) was incorporated to provide a transparent electrode. Also, an AZO layer was taken to be sandwiched between the 2D PCBR and the stack of nine semiconductor layers in order to avoid the deterioration of the electrical properties of the a-Si alloy closest to the metal [33], which was chosen to be silver [34]. The total absorptance and the useful absorptance calculated using the rigorous coupled-wave approach (RCWA) [35, 36, 24] were correlated against the predicted excitations of GWMs.

The plan of this paper is as follows. Section 2 is divided into four parts. Section 2.1 presents the boundary-value problem that can be solved to determine the optical electromagnetic fields everywhere in a device comprising a stratified, isotropic dielectric material atop a 2D PCBR, when the device is illuminated by a plane wave. The formulations for useful and total absorptances are discussed in Sec. 2.2. Section 2.3 provides brief descriptions of the underlying canonical problems to predict the excitation of SPP waves and WGMs. Excitation of GWMs is discussed in Sec. 2.4. Section 3 is divided into two parts. The wavenumbers of the predicted GWMs are presented in Sec. 3.1. Correlations of the absorptances with the predicted GWMs are discussed in Sec. 3.2. The paper concludes with some remarks in Sec. 4.

An $\exp (-i \omega t)$ dependence on time $t$ is implicit, with $\omega$ denoting the angular frequency and $i=\sqrt{-1}$. The free-space wavenumber, the free-space wavelength, and the intrinsic impedance of free space are denoted by $k_{0}=\omega \sqrt{\mu_{0} \varepsilon_{0}}, \lambda_{0}=2 \pi / k_{0}$, and $\eta_{0}=\sqrt{\mu_{0} / \varepsilon_{0}}$, respectively, with $\mu_{0}$ being the permeability and $\varepsilon_{0}$ the permittivity of free space. Vectors are underlined; the Cartesian unit vectors are identified as $\underline{\hat{u}}_{x}$, $\underline{\hat{u}}_{y}$, and $\underline{\hat{u}}_{z}$; and column vectors as well as matrixes are in boldface.

\section{Theory in Brief}

\subsection{Boundary-value problem for tandem solar cell}

Let us consider the boundary-value problem shown in Fig. 1 for a tandem solar cell containing three $p$ $i$ - $n$ junctions. The solar cell occupies the region $\mathcal{X}:\left\{(x, y, z) \mid-\infty<x<\infty,-\infty<y<\infty, 0<z<L_{\mathrm{t}}\right\}$, with the half spaces $z<0$ and $z>L_{\mathrm{t}}$ occupied by air. The reference unit cell is identified as $\mathcal{R}$ : $\left\{(x, y, z) \mid-L_{\mathrm{x}} / 2<x<L_{\mathrm{x}} / 2,-L_{\mathrm{y}} / 2<y<L_{\mathrm{y}} / 2,0<z<L_{\mathrm{t}}\right\}$, the backreflector being periodic along both the $x$ and $y$ axes.

The region $0<z<L_{\mathrm{d}}=L_{\mathrm{w}}+L_{\mathrm{s}}+L_{\mathrm{a}}$ is occupied by a cascade of homogeneous layers and is compactly characterized by the permittivity $\varepsilon_{\mathrm{d}}\left(z, \lambda_{0}\right)$, which is a piecewise constant function of $z$. The top layer $0<z<$ $L_{\mathrm{w}}$ and the bottom layer $L_{\mathrm{w}}+L_{\mathrm{s}}<z<L_{\mathrm{d}}$ are made of AZO with permittivity $\varepsilon_{\mathrm{w}}\left(\lambda_{0}\right)$. The semiconductor layers in the region $L_{\mathrm{w}}<z<L_{\mathrm{w}}+L_{\mathrm{s}}$ are identified in Fig. 1(b). The region $L_{\mathrm{d}}+L_{\mathrm{g}}<z<L_{\mathrm{d}}+L_{\mathrm{g}}+L_{\mathrm{m}}$ is occupied by a metal with permittivity $\varepsilon_{\mathrm{m}}\left(\lambda_{0}\right)$.

The region $L_{\mathrm{d}}<z<L_{\mathrm{d}}+L_{\mathrm{g}}$, henceforth termed the grating region, contains a periodically undulating surface with period $L_{\mathrm{x}}$ along the $x$ axis and period $L_{\mathrm{y}}$ along the $y$ axis. In the grating region, $\mathcal{X}$ possesses rectangular symmetry in the $x y$ plane. The permittivity $\varepsilon_{\mathrm{g}}\left(x, y, z, \lambda_{0}\right)$ in the grating region can be stated as

$$
\begin{gathered}
\varepsilon_{\mathrm{g}}\left(x, y, z, \lambda_{0}\right)=\varepsilon_{\mathrm{m}}\left(\lambda_{0}\right)+\left[\varepsilon_{\mathrm{w}}\left(\lambda_{0}\right)-\varepsilon_{\mathrm{m}}\left(\lambda_{0}\right)\right] \mathcal{U}\left[z-g_{1}(x)\right] \mathcal{U}\left[z-g_{2}(y)\right], \\
|x|<\zeta_{\mathrm{x}} L_{\mathrm{x}} / 2, \quad|y|<\zeta_{\mathrm{y}} L_{\mathrm{y}} / 2, \quad z \in\left(L_{\mathrm{d}}, L_{\mathrm{d}}+L_{\mathrm{g}}\right),
\end{gathered}
$$

where the unit step function

$$
\mathcal{U}(\sigma)= \begin{cases}0, & \sigma<0 \\ 1, & \sigma \geq 0\end{cases}
$$




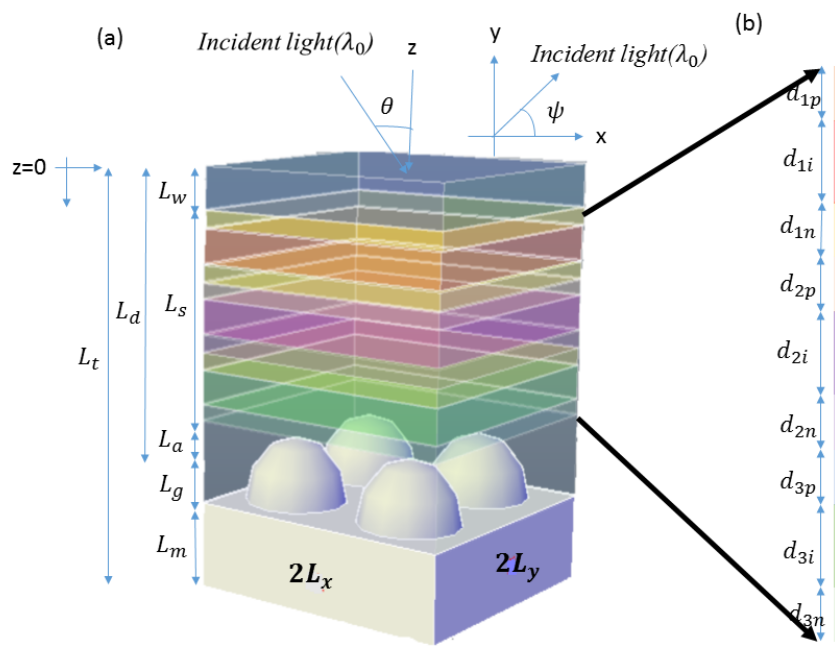

Figure 1: (a) Schematic of the tandem solar cell comprising three $p$ - $i$ - $n$ junctions of a-Si alloys on a 2D PCBR. The wavevector of the incident plane wave is inclined at angle $\theta$ with respect to the $z$ axis and angle $\psi$ with respect to the $x$ axis in the $x y$ plane. (b) Nine semiconductors layers of the three $p-i-n$ junctions.

and $\zeta_{\mathrm{x}} \in[0,1]$ as well as $\zeta_{\mathrm{y}} \in[0,1]$ are the duty cycles. We chose the grating-shape functions

$$
g_{1}(x)= \begin{cases}L_{\mathrm{d}}+L_{\mathrm{g}}\left[1-\cos \left(2 \pi \frac{\pi x}{\zeta_{\mathrm{x}} L_{\mathrm{x}}}\right)\right], & x \in\left[-\frac{\zeta_{\mathrm{x}} L_{\mathrm{x}}}{2}, \frac{\zeta_{\mathrm{x}} L_{\mathrm{x}}}{2}\right], \\ L_{\mathrm{d}}+L_{\mathrm{g}}, & x \notin\left[-\frac{\zeta_{\mathrm{x}} L_{\mathrm{x}}}{2}, \frac{\zeta_{\mathrm{x}} L_{\mathrm{x}}}{2}\right],\end{cases}
$$

and

$$
g_{2}(y)= \begin{cases}L_{\mathrm{d}}+L_{\mathrm{g}}\left[1-\cos \left(2 \pi \frac{\pi y}{\zeta_{\mathrm{y}} L_{\mathrm{y}}}\right)\right], & y \in\left[-\frac{\zeta_{\mathrm{y}} L_{\mathrm{y}}}{2}, \frac{\zeta_{\mathrm{y}} \zeta_{\mathrm{y}}}{2}\right], \\ L_{\mathrm{d}}+L_{\mathrm{g}}, & y \notin\left[-\frac{\zeta_{\mathrm{y}} L_{\mathrm{y}}}{2}, \frac{\zeta_{\mathrm{y}} L_{\mathrm{y}}}{2}\right],\end{cases}
$$

to represent hillocks for all data reported in this paper. The grating-shape functions chosen here are only for illustration, many other choices fit for experimental study being also available [13].

Suppose that an arbitrarily polarized plane wave, propagating in the half space $z<0$ at an angle $\theta \in\left[0^{\circ}, 90^{\circ}\right)$ with respect to the $z$ axis and an angle $\psi \in\left[0^{\circ}, 360^{\circ}\right)$ with respect to the $x$ axis in the $x y$ plane, is incident on the plane $z=0$. The electric field phasor of this plane wave can be stated as

$$
\underline{E}_{\mathrm{inc}}(\underline{r})=\left(\bar{a}_{\mathrm{s}} \underline{s}^{(0,0)}+\bar{a}_{\mathrm{p}} \underline{p}_{+}^{(0,0)}\right) \exp \left[i\left(\underline{\kappa}^{(0,0)}+\alpha_{0}^{(0,0)} \underline{\hat{u}}_{z}\right) \cdot \underline{r}\right],
$$

where $\bar{a}_{\mathrm{s}}$ and $\bar{a}_{\mathrm{p}}$ are the known coefficients of $s$ - and $p$-polarized components, respectively. Here and hereafter, 
the following quantities are used:

$$
\left.\begin{array}{l}
\underline{\kappa}^{(m, n)}=k_{\mathrm{x}}^{(m)} \underline{\hat{u}}_{x}+k_{\mathrm{y}}^{(n)} \underline{\underline{u}}_{y} \\
k_{\mathrm{x}}^{(m)}=k_{0} \sin \theta \cos \psi+m\left(2 \pi / L_{\mathrm{x}}\right) \\
k_{\mathrm{y}}^{(n)}=k_{0} \sin \theta \sin \psi+n\left(2 \pi / L_{\mathrm{y}}\right) \\
k_{\mathrm{xy}}^{(m, n)}=+\sqrt{\underline{\kappa}^{(m, n)} \cdot \underline{\kappa}^{(m, n)}} \\
\alpha_{0}^{(m, n)}=+\sqrt{k_{0}^{2}-\underline{\kappa}^{(m, n)} \cdot \underline{\kappa}^{(m, n)}} \\
\underline{s}^{(m, n)}=-\frac{k_{\mathrm{y}}^{(n)}}{k_{\mathrm{xy}}^{(m, n)}} \underline{\hat{u}}_{x}+\frac{k_{\mathrm{x}}^{(m)}}{k_{\mathrm{xy}}^{(m, n)}} \underline{\hat{u}}_{y} \\
\underline{p}_{+}^{(m, n)}=-\left(\frac{k_{\mathrm{x}}^{(m)}}{k_{\mathrm{xy}}^{(m, n)}} \underline{\hat{u}}_{x}+\frac{k_{\mathrm{y}}^{(n)}}{k_{\mathrm{xy}}^{(m, n)}} \underline{\hat{u}}_{y}\right) \frac{\alpha_{0}^{(m, n)}}{k_{0}}+\frac{k_{\mathrm{xy}}^{(m, n)}}{k_{0}} \underline{\hat{u}}_{z} \\
\underline{p}_{-}^{(m, n)}=\left(\frac{k_{\mathrm{x}}^{(m)}}{k_{\mathrm{xy}}^{(m, n)}} \hat{\underline{u}}_{x}+\frac{k_{\mathrm{y}}^{(n)}}{k_{\mathrm{xy}}^{(m, n)}} \underline{\hat{u}}_{y}\right) \frac{\alpha_{0}^{(m, n)}}{k_{0}}+\frac{k_{\mathrm{xy}}^{(m, n)}}{k_{0}} \underline{\hat{u}}_{z}
\end{array}\right\}
$$

As a result of the metallic PCBR being doubly periodic, the $x$ - and $y$-dependences of the electric and magnetic field phasors are represented everywhere as an infinite series of Floquet harmonics as [35, 36, 24]

$$
\left.\begin{array}{l}
\underline{E}(x, y, z)=\sum_{m \in \mathbb{Z}} \sum_{n \in \mathbb{Z}} \underline{e}^{(m, n)}(z) \exp \left(i \underline{\kappa}^{(m, n)} \cdot \underline{r}\right) \\
\underline{H}(x, y, z)=\sum_{m \in \mathbb{Z}} \sum_{n \in \mathbb{Z}} \underline{h}^{(m, n)}(z) \exp \left(i \underline{\kappa}^{(m, n)} \cdot \underline{r}\right)
\end{array}\right\},
$$

where

$$
\left.\begin{array}{l}
\underline{e}^{(m, n)}(z)=e_{x}^{(m, n)}(z) \underline{\hat{u}}_{x}+e_{y}^{(m, n)}(z) \underline{\hat{u}}_{y}+e_{z}^{(m, n)}(z) \underline{\hat{u}}_{z} \\
\underline{h}^{(m, n)}(z)=h_{x}^{(m, n)}(z) \underline{\hat{\hat{x}}}_{x}+h_{y}^{(m, n)}(z) \underline{\hat{\hat{u}}}_{y}+h_{z}^{(m, n)}(z) \underline{\hat{\underline{u}}}_{z}
\end{array}\right\}
$$

are expansion coefficients. Accordingly, the incident and the reflected electric field phasors are represented as

$$
\left.\begin{array}{l}
\underline{E}_{\mathrm{inc}}(x, y, z)=\sum_{m \in \mathbb{Z}} \sum_{n \in \mathbb{Z}}\left\{\left(a_{\mathrm{s}}^{(m, n)} \underline{s}^{(m, n)}+a_{\mathrm{p}}^{(m, n)} \underline{p}_{+}^{(m, n)}\right)\right. \\
\left.\quad \times \exp \left[i\left(\underline{\kappa}^{(m, n)}+\alpha_{0}^{(m, n)} \underline{\hat{u}}_{z}\right) \cdot \underline{r}\right]\right\} \\
\underline{E}_{\mathrm{ref}}(x, y, z)=\sum_{m \in \mathbb{Z}} \sum_{n \in \mathbb{Z}}\left\{\left(r_{\mathrm{s}}^{(m, n)} \underline{s}^{(m, n)}+r_{\mathrm{p}}^{(m, n)} \underline{p}_{-}^{(m, n)}\right)\right. \\
\left.\quad \times \exp \left[i\left(\underline{\kappa}^{(m, n)}-\alpha_{0}^{(m, n)} \underline{\hat{u}}_{z}\right) \cdot \underline{r}\right]\right\}
\end{array}\right\}, \quad z<0,
$$

and the transmitted electric field phasor as

$$
\begin{aligned}
\underline{E}_{\mathrm{tr}}(x, y, z)=\sum_{m \in \mathbb{Z}} & \sum_{n \in \mathbb{Z}}\left\{\left(t_{\mathrm{s}}^{(m, n)} \underline{s}^{(m, n)}+t_{\mathrm{p}}^{(m, n)} \underline{p}_{+}^{(m, n)}\right)\right. \\
& \left.\times \exp \left[i\left(\underline{\kappa}^{(m, n)}+\alpha_{0}^{(m, n)} \underline{\hat{u}}_{z}\right) \cdot\left(\underline{r}-L_{\mathrm{t}} \underline{\hat{\underline{u}}}_{z}\right)\right]\right\}, \quad z>L_{\mathrm{t}},
\end{aligned}
$$

where the coefficients $a_{\mathrm{s}}^{(m, n)}=\bar{a}_{\mathrm{s}} \delta_{m 0} \delta_{n 0}$ and $a_{\mathrm{p}}^{(m, n)}=\bar{a}_{\mathrm{p}} \delta_{m 0} \delta_{n 0}$ in Eq. (9) ${ }_{1}$ are known with $\delta_{m m^{\prime}}$ denoting the Kronecker delta, but the coefficients $r_{\mathrm{s}}^{(m, n)}, r_{\mathrm{p}}^{(m, n)}, t_{\mathrm{s}}^{(m, n)}$, and $t_{\mathrm{p}}^{(m, n)}$ in Eq. $(9)_{2}$ and Eq. (10) have to be determined. Finally, the permittivity $\varepsilon(x, y, z)$ everywhere is represented by the Fourier series

$$
\varepsilon(x, y, z)=\sum_{m \in \mathbb{Z}} \sum_{n \in \mathbb{Z}} \varepsilon^{(m, n)}(z) \exp \left[i\left(\underline{\kappa}^{(m, n)}-\underline{\kappa}^{(0,0)}\right) \cdot \underline{r}\right]
$$


where $\varepsilon^{(m, n)}(z)$ are Fourier coefficients.

Computational tractability requires the expansions in Eqs. (7)-(11) to be truncated to include only $m \in\left\{-M_{t}, \ldots, M_{t}\right\}$ and $n \in\left\{-N_{t}, \ldots, N_{t}\right\}$, with $M_{t} \geq 0$ and $N_{t} \geq 0$. Furthermore, a superindex

$$
\tau=m\left(2 N_{t}+1\right)+n, \quad m \in\left[-M_{t}, M_{t}\right], \quad n \in\left[-N_{t}, N_{t}\right],
$$

is defined for convenience. Then, $\tau \in\left[-\tau_{t}, \tau_{t}\right]$, where $\tau_{t}=2 M_{t} N_{t}+M_{t}+N_{t}$. Also, both the mapping from $(m, n)$ to $\tau$ and the inverse mapping from $\tau$ to $(m, n)$ are injective [37]. Thereafter, column vectors

$$
\left.\begin{array}{l}
\breve{\mathbf{e}}_{\sigma}(z)=\left[e_{\sigma}^{\left(-\tau_{t}\right)}(z), e_{\sigma}^{\left(-\tau_{t}+1\right)}(z), \ldots, e_{\sigma}^{\left(\tau_{t}-1\right)}(z), e_{\sigma}^{\left(\tau_{t}\right)}(z)\right]^{T} \\
\breve{\mathbf{h}}_{\sigma}(z)=\left[h_{\sigma}^{\left(-\tau_{t}\right)}(z), h_{\sigma}^{\left(-\tau_{t}+1\right)}(z), \ldots, h_{\sigma}^{\left(\tau_{t}-1\right)}(z), h_{\sigma}^{\left(\tau_{t}\right)}(z)\right]^{T}
\end{array}\right\}, \quad \sigma \in\{x, y, z\},
$$

of length $2 \tau_{t}+1$ are set up, the superscript $T$ denoting the transpose. The Toeplitz matrix [38]

$$
\breve{\boldsymbol{\varepsilon}}(z)=\left[\begin{array}{ccccc}
\breve{\varepsilon}^{\left(-\tau_{t},-\tau_{t}\right)}(z) & \breve{\varepsilon}^{\left(-\tau_{t},-\tau_{t}+1\right)}(z) & \ldots & \breve{\varepsilon}^{\left(-\tau_{t}, \tau_{t}-1\right)}(z) & \breve{\varepsilon}^{\left(-\tau_{t}, \tau_{t}\right)}(z) \\
\breve{\varepsilon}^{\left(-\tau_{t}+1,-\tau_{t}\right)}(z) & \breve{\varepsilon}^{\left(-\tau_{t}+1,-\tau_{t}+1\right)}(z) & \ldots & \breve{\varepsilon}^{\left(-\tau_{t}+1, \tau_{t}-1\right)}(z) & \breve{\varepsilon}^{\left(-\tau_{t}+1, \tau_{t}\right)}(z) \\
\ldots & \ldots & \ldots & \ldots & \ldots \\
\breve{\varepsilon}^{\left(\tau_{t}-1,-\tau_{t}\right)}(z) & \breve{\varepsilon}^{\left(\tau_{t}-1,-\tau_{t}+1\right)}(z) & \ldots & \breve{\varepsilon}^{\left(\tau_{t}-1, \tau_{t}-1\right)}(z) & \breve{\varepsilon}^{\left(\tau_{t}-1, \tau_{t}\right)}(z) \\
\breve{\varepsilon}^{\left(\tau_{t},-\tau_{t}\right)}(z) & \breve{\varepsilon}^{\left(\tau_{t},-\tau_{t}+1\right)}(z) & \ldots & \breve{\varepsilon}^{\left(\tau_{t}, \tau_{t}-1\right)}(z) & \breve{\varepsilon}^{\left(\tau_{t}, \tau_{t}\right)}(z)
\end{array}\right] .
$$

contains the Fourier coefficients appearing in Eq. (8) with $\breve{\varepsilon}^{\left(\tau, \tau^{\prime}\right)}(z)=\varepsilon^{\left(m-m^{\prime}, n-n^{\prime}\right)}(z)$. Finally, the $\left(2 \tau_{t}+\right.$ 1) $\times\left(2 \tau_{t}+1\right)$ Fourier-wavenumber matrixes

$$
\left.\begin{array}{l}
\breve{\mathbf{K}}_{\mathrm{x}}=\operatorname{diag}\left[\breve{k}_{\mathrm{x}}^{\left(-\tau_{t}\right)}, \breve{k}_{\mathrm{x}}^{\left(-\tau_{t}+1\right)}, \ldots, \breve{k}_{\mathrm{x}}^{\left(\tau_{t}-1\right)}, \breve{k}_{\mathrm{x}}^{\left(\tau_{t}\right)}\right] \\
\breve{\mathbf{K}}_{\mathrm{y}}=\operatorname{diag}\left[\breve{k}_{\mathrm{y}}^{\left(-\tau_{t}\right)}, \breve{k}_{\mathrm{y}}^{\left(-\tau_{t}+1\right)}, \ldots, \breve{k}_{\mathrm{y}}^{\left(\tau_{t}-1\right)}, \breve{k}_{\mathrm{y}}^{\left(\tau_{t}\right)}\right]
\end{array}\right\}
$$

are set up with $\breve{k}_{\mathrm{x}}^{(\tau)}=k_{\mathrm{x}}^{(m)}$ and $\breve{k}_{\mathrm{y}}^{(\tau)}=k_{\mathrm{y}}^{(n)}$.

The frequency-domain Maxwell curl postulates yield the matrix ordinary differential equation [24]

$$
\frac{d}{d z} \breve{\mathbf{f}}(z)=i \breve{\mathbf{P}}(z) \cdot \breve{\mathbf{f}}(z),
$$

where the $4\left(2 \tau_{t}+1\right)$-column vector

$$
\breve{\mathbf{f}}(z)=\left[\begin{array}{c}
\breve{\mathbf{e}}_{x}(z) \\
\breve{\mathbf{e}}_{y}(z) \\
\breve{\mathbf{h}}_{x}(z) \\
\breve{\mathbf{h}}_{y}(z)
\end{array}\right]
$$

and the $4\left(2 \tau_{t}+1\right) \times 4\left(2 \tau_{t}+1\right)$ matrix

$$
\begin{gathered}
\breve{\mathbf{P}}(z)=\omega\left[\begin{array}{cccc}
\breve{\mathbf{0}} & \breve{\mathbf{0}} & \breve{\mathbf{0}} & \mu_{0} \breve{\mathbf{I}} \\
\breve{\mathbf{0}} & \breve{\mathbf{0}} & -\mu_{0} \breve{\mathbf{I}} & \breve{\mathbf{0}} \\
\breve{\mathbf{0}} & -\breve{\boldsymbol{\varepsilon}}(z) & \breve{\mathbf{0}} & \breve{\mathbf{0}} \\
\breve{\varepsilon}(z) & \breve{\mathbf{0}} & \breve{\mathbf{0}} & \breve{\mathbf{0}}
\end{array}\right] \\
+\frac{1}{\omega}\left[\begin{array}{ccccc}
\breve{\mathbf{0}} & \breve{\mathbf{0}} & \breve{\mathbf{K}}_{x} \cdot[\breve{\boldsymbol{\varepsilon}}(z)]^{-1} \cdot \breve{\mathbf{K}}_{y} & -\breve{\mathbf{K}}_{x} \cdot[\breve{\varepsilon}(z)]^{-1} \cdot \breve{\mathbf{K}}_{x} \\
\breve{\mathbf{0}} & \breve{\mathbf{0}} & \breve{\mathbf{K}}_{y} \cdot[\breve{\boldsymbol{\varepsilon}}(z)]^{-1} \cdot \breve{\mathbf{K}}_{y} & -\breve{\mathbf{K}}_{y} \cdot[\breve{\boldsymbol{\varepsilon}}(z)]^{-1} \cdot \breve{\mathbf{K}}_{x} \\
-\mu_{0}^{-1} \breve{\mathbf{K}}_{x} \cdot \breve{\mathbf{K}}_{y} & \mu_{0}^{-1} \breve{\mathbf{K}}_{x} \cdot \breve{\mathbf{K}}_{x} & \breve{\mathbf{0}} & \breve{\mathbf{0}} \\
-\mu_{0}^{-1} \breve{\mathbf{K}}_{y} \cdot \breve{\mathbf{K}}_{y} & \mu_{0}^{-1} \breve{\mathbf{K}}_{y} \cdot \breve{\mathbf{K}}_{x} & \breve{\mathbf{0}} & \breve{\mathbf{0}}
\end{array}\right]
\end{gathered}
$$


contains $\breve{\mathbf{0}}$ as the $\left(2 \tau_{t}+1\right) \times\left(2 \tau_{t}+1\right)$ null matrix and $\breve{\mathbf{I}}$ as the $\left(2 \tau_{t}+1\right) \times\left(2 \tau_{t}+1\right)$ identity matrix.

In order to solve Eq. (16), the region $\mathcal{R}$ is partitioned into a sufficiently large number of thin slices along the $z$ direction [24]. Each slice is taken to be homogeneous along the $z$ axis but it is either homogeneous or periodically nonhomogeneous along the $x$ and $y$ axes; thus, $\breve{\mathbf{P}}(z)$ is assumed to be uniform in each slice. Boundary conditions are enforced on the planes $z=0$ and $z=L_{\mathrm{t}}$ to match the fields to the incident, reflected, and transmitted waves, as appropriate. A stable numerical marching algorithm is then used to determine the Fourier coefficients of the electric and magnetic field phasors in each slice[24]. Finally, the $z$ components of the electric and magnetic field phasors in the device can be obtained through $\breve{\mathbf{e}}_{z}(z)=-[\omega \boldsymbol{\varepsilon}(z)]^{-1} \cdot\left[\breve{\mathbf{K}}_{x} \cdot \breve{\mathbf{h}}_{y}(z)-\right.$ $\left.\breve{\mathbf{K}}_{y} \cdot \breve{\mathbf{h}}_{x}(z)\right]$ and $\breve{\mathbf{h}}_{z}(z)=\left(\omega \mu_{0}\right)^{-1}\left[\breve{\mathbf{K}}_{x} \cdot \breve{\mathbf{e}}_{y}(z)-\breve{\mathbf{K}}_{y} \cdot \breve{\mathbf{e}}_{x}(z)\right]$. Thus, the electric field phasor can be determined everywhere. The entire procedure was implemented on the Mathematica ${ }^{\circledR}$ platform.

\subsection{Total and useful absorptances}

At any location inside the device, the absorption rate of the monochromatic optical energy per unit volume is given by

$$
Q(x, y, z)=\frac{1}{2} \omega \operatorname{Im}\{\varepsilon(x, y, z)\}|\underline{E}(x, y, z)|^{2} .
$$

The useful absorptance [39]

$$
\bar{A}^{\mathrm{sc}}=\frac{2 \eta_{0}}{L_{\mathrm{x}} L_{\mathrm{y}}\left(\left|\bar{a}_{\mathrm{s}}\right|^{2}+\left|\bar{a}_{\mathrm{p}}\right|^{2}\right) \cos \theta} \iiint_{\mathcal{R}_{\mathrm{sc}}} Q(x, y, z) d x d y d z
$$

is calculated by integrating $Q(x, y, z)$ over the region $\mathcal{R}_{\text {sc }} \subset \mathcal{R}$ occupied by the semiconductor layers. Likewise, absorptance in the metal is given by

$$
\bar{A}^{\mathrm{met}}=\frac{2 \eta_{\mathrm{o}}}{L_{\mathrm{x}} L_{\mathrm{y}}\left(\left|\bar{a}_{\mathrm{s}}\right|^{2}+\left|\bar{a}_{\mathrm{p}}\right|^{2}\right) \cos \theta} \iiint_{\mathcal{R}_{\mathrm{met}}} Q(x, y, z) d x d y d z,
$$

where $\mathcal{R}_{\text {met }} \subset \mathcal{R}$ is the region occupied by the metal. The total absorptance is then the sum

$$
\bar{A}^{\mathrm{tot}}=\bar{A}^{\mathrm{sc}}+\bar{A}^{\mathrm{met}},
$$

if $\varepsilon_{\mathrm{w}}$ is purely real.

Four reflection and four transmission coefficients of order $(m, n)$ are defined as the elements in the $2 \times 2$ matrices appearing in the following relations [24]:

$$
\left[\begin{array}{l}
r_{\mathrm{s}}^{(m, n)} \\
r_{\mathrm{p}}^{(m, n)}
\end{array}\right]=\left[\begin{array}{ll}
r_{\mathrm{ss}}^{(m, n)} & r_{\mathrm{sp}}^{(m, n)} \\
r_{\mathrm{ps}}^{(m, n)} & r_{\mathrm{pp}}^{(m, n)}
\end{array}\right] \cdot\left[\begin{array}{l}
\bar{a}_{\mathrm{s}} \\
\bar{a}_{\mathrm{p}}
\end{array}\right], \quad\left[\begin{array}{c}
t_{\mathrm{s}}^{(m, n)} \\
t_{\mathrm{p}}^{(m, n)}
\end{array}\right]=\left[\begin{array}{cc}
t_{\mathrm{ss}}^{(m, n)} & t_{\mathrm{sp}}^{(m, n)} \\
t_{\mathrm{ps}}^{(m, n)} & t_{\mathrm{pp}}^{(m, n)}
\end{array}\right] \cdot\left[\begin{array}{l}
\bar{a}_{\mathrm{s}} \\
\bar{a}_{\mathrm{p}}
\end{array}\right] .
$$

Coefficients of order $(0,0)$ are classified as specular, whereas coefficients of all other orders are nonspecular. Four reflectances and four linear transmittances of order $(m, n)$ are defined as

$$
R_{\mathrm{sp}}^{(m, n)}=\frac{\operatorname{Re}\left[\alpha_{0}^{(m, n)}\right]}{\alpha_{0}^{(0,0)}}\left|r_{\mathrm{sp}}^{(m, n)}\right|^{2} \in[0,1]
$$

etc., and two absorptances as

$$
\left.\begin{array}{l}
A_{\mathrm{s}}=1-\sum_{m=-M_{t}} \sum_{n=-N_{t}}^{m=M_{t}}\left(R_{\mathrm{ss}}^{(m, n)}+R_{\mathrm{ps}}^{(m, n)}+T_{\mathrm{ss}}^{(m, n)}+T_{\mathrm{ps}}^{(m, n)}\right) \in[0,1] \\
A_{\mathrm{p}}=1-\sum_{m=-M_{t}} \sum_{n=-N_{t}}^{m=M_{t}}\left(R_{\mathrm{pp}}^{(m, n)}+R_{\mathrm{sp}}^{(m, n)}+T_{\mathrm{pp}}^{(m, n)}+T_{\mathrm{sp}}^{(m, n)}\right) \in[0,1]
\end{array}\right\} .
$$


These are total absorptances in that they contain the contributions of the semiconductors and the metal in the solar cell. Whereas $\bar{A}^{\text {tot }}, \bar{A}^{\text {sc }}$, and $\bar{A}^{\text {met }}$ are defined for incident light of arbitrary polarization state, $A_{\mathrm{s}}$ is defined for incident $s$-polarized light and $A_{\mathrm{p}}$ for incident $p$-polarized light. All absorptances presented in Sec. 3 were calculated for a solar cell comprising just one triple $p-i$ - $n$ junction, as shown in Fig. 1.

\subsection{Canonical boundary-value problems}

Two separate canonical boundary-value problems were solved to correlate peaks in the spectrums of various absorptances with the excitation of SPP waves and WGMs. Details on both canonical problems are available elsewhere [39] for the interested reader, but we note the following salient features of both canonical problems.

\subsubsection{SPP waves}

The complex-valued wavenumbers $q \neq 0$ of SPP waves for a specific value of $\lambda_{0}$ were obtained by solving a canonical boundary-value problem [24, 29], with the assumptions that the backreflector metal occupies the half space $z<0$, a periodically semi-infinite cascade of three $p$ - $i$ - $n$ junctions occupies the half space $z>0$, and there are no AZO layers.

\subsubsection{Waveguide modes}

An open-faced waveguide is formed by the three $p-i$ - $n$ junctions interposed between two half spaces, one occupied by air and the other by the backreflector metal of thickness considerably exceeding the skin depth [40]. For a specific value of $\lambda_{0}$, this waveguide can support the propagation of multiple WGMs (with wavenumbers $q \neq 0$ ) which can play significant light-trapping roles [21, 22, 23]. We ignored the AZO layers for this canonical problem as well.

\subsection{Excitation of SPP waves and WGMs}

Planewave illumination will excite a GWM of wavenumber $q$ as a Floquet harmonic of order $(m, n)$, provided that [24]

$$
\pm \operatorname{Re}[q] \simeq k_{\mathrm{xy}}^{(m, n)}
$$

When $L_{\mathrm{x}}=L_{\mathrm{y}}=L$, the right side of Eq. (26) simplifies to yield

$$
\pm \operatorname{Re}\left[q / k_{0}\right] \simeq\left\{\left[\sin \theta+(m \cos \psi+n \sin \psi)\left(\lambda_{0} / L\right)\right]^{2}+\left[(m \sin \psi-n \cos \psi)\left(\lambda_{0} / L\right)\right]^{2}\right\}^{\frac{1}{2}}
$$

Since the thickness $L_{\mathrm{d}}$ is finite, shifts in the predictions of $\theta$ for specific values of $\lambda_{0}$ and $\psi$ are possible for SPP waves. Also, shifts are possible for both SPP waves and WGMs, because both canonical problems were formulated and solved with $L_{\mathrm{w}}=L_{\mathrm{a}}=0$. Finally, shifts can also be due to $L_{\mathrm{g}} \neq 0$ [41]. Therefore, for all absorptance spectrums presented in this paper, we accepted predictions of $\theta$ from Eq. (27) with $\pm 1^{\circ}$ tolerance. However, let us note that not every possible GWM is strongly excited by planewave illumination.

Finally, it is important to note that depolarization can occur because the PCBR is doubly periodic. Accordingly, illumination by a linearly polarized plane wave for a specific value of $\psi$ can excite a GWM of a different polarization state propagating in a direction specified by the angle $\varphi$ that may differ from $\psi$ $[21,42]$.

\section{Numerical results and discussion}

All optical and geometric parameters were chosen only to illustrate the relationships of the WGMs to total and useful absorptances, but still are representative of actual tandem solar cells [30]. The compositions, bangaps, and thicknesses of the nine hydrogenated a-Si alloys for the nine semiconductor layers are presented in Table I. 
The permittivity of each alloy was calculated as a function of $\lambda_{0}$, using a model provided by Ferlauto et al. $[18,32]$. The spectrums of all nine permittivities, normalized by $\varepsilon_{0}$, are plotted in Fig. 2. The 2D PCBR was taken to be made of silver [34]. The refractive index of AZO was taken as a function of $\lambda_{0}$ from Gao et al. [43].

Table I: Compositions, bandgaps, and thicknesses of hydrogenated a-Si alloys used for the nine semiconductor layers in the triple- $p$ - $i$ - $n$-junction tandem solar cell.

\begin{tabular}{llll}
\hline \hline Layer & Composition & Bandgap (eV) & Thickness (nm) \\
\hline $1 p$ & $\mathrm{a}-\mathrm{Si} i_{1-u} \mathrm{C}_{u}: \mathrm{H}$ & 1.95 & 20 \\
$1 i$ & $\mathrm{a}-\mathrm{Si}: \mathrm{H}$ & 1.8 & 200 \\
$1 n$ & $\mathrm{a}-\mathrm{Si}: \mathrm{H}$ & 1.8 & 20 \\
\hline $2 p$ & $\mathrm{a}-\mathrm{Si}_{1-u} \mathrm{C}_{u}: \mathrm{H}$ & 1.95 & 20 \\
$2 i$ & $\mathrm{a}-\mathrm{Si}_{1-u} \mathrm{Ge}_{u}: \mathrm{H}$ & 1.58 & 200 \\
$2 n$ & $\mathrm{a}-\mathrm{Si}: \mathrm{H}$ & 1.8 & 20 \\
\hline $3 p$ & $\mathrm{a}-\mathrm{Si}: \mathrm{H}$ & 1.8 & 20 \\
$3 i$ & $\mathrm{a}-\mathrm{Si} i_{1-u} \mathrm{Ge}_{u}: \mathrm{H}$ & 1.39 & 200 \\
$3 n$ & $\mathrm{a}-\mathrm{Si}: \mathrm{H}$ & 1.8 & 20 \\
\hline \hline
\end{tabular}
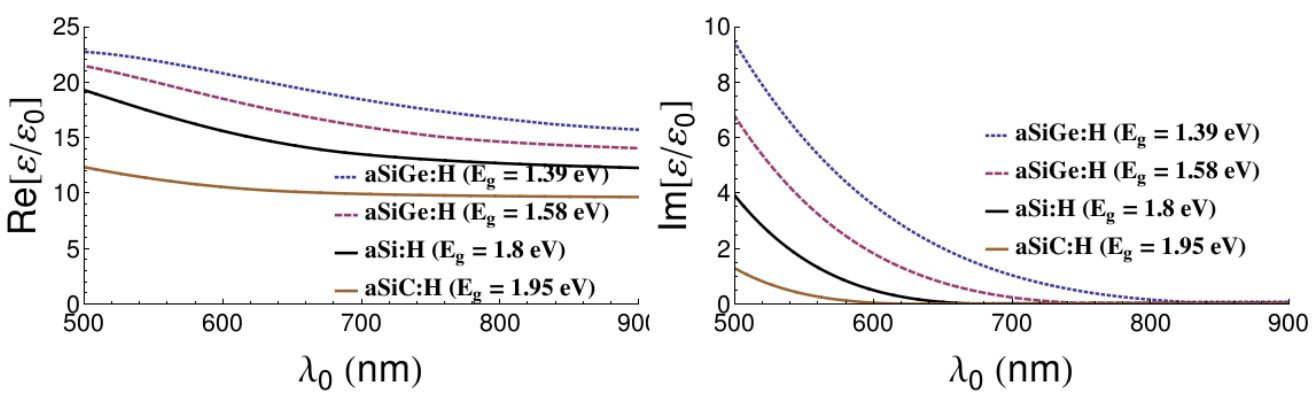

Figure 2: Spectrums of the relative permittivity $\varepsilon / \varepsilon_{0}$ of the different semiconductor alloys used in the triple- $p$ - $i$ - $n$-junction tandem solar cell.

The following dimensions were chosen: $L_{\mathrm{w}}=100 \mathrm{~nm}, L_{\mathrm{a}}=60 \mathrm{~nm}, L_{\mathrm{g}}=80 \mathrm{~nm}, L_{\mathrm{m}}=30 \mathrm{~nm}$, $L_{\mathrm{x}}=L_{\mathrm{y}}=400 \mathrm{~nm}$, and $\zeta_{\mathrm{x}}=\zeta_{\mathrm{y}}=1$. We used $M_{t}=N_{t}$ accordingly. Furthermore, we used $M_{t} \leq 12$, which ensured the convergence of all non-zero reflectances and absorptances to within $\pm 1 \%$ for every $\lambda_{0} \in$ $\{500,502, \ldots, 898,900\} \mathrm{nm}$. Here, convergence was defined to have occurred when there was a difference not exceeding $1 \%$ in magnitude between the results for $M_{t}=N-1$ and $M_{t}=N$. Higher values of $M_{t}$ were found to be necessary for higher $\lambda_{0}$ as the chosen semiconductor alloys are then less absorbing and the effect of grating is more pronounced.

\subsection{Prediction of GWM wavenumbers}

The real parts of the normalized wavenumbers $q / k_{0}$ of SPP waves are presented in Fig 3 as functions of $\lambda_{0} \in\{500,501, \ldots, 899,900\} \mathrm{nm}$. These wavenumbers are organized into three branches (labeled $\left.s 1-s 3\right)$ for $s$-polarized SPP waves and seven branches (labeled $p 1-p 7$ ) for $p$-polarized SPP waves. The real parts of the normalized wavenumbers $q / k_{0}$ of the WGMs are presented in Fig 4 . These wavenumbers are arranged into six branches for both $s$ - and $p$-polarized WGMs labeled $s 1-s 6$ and $p 1-p 6$, respectively. 

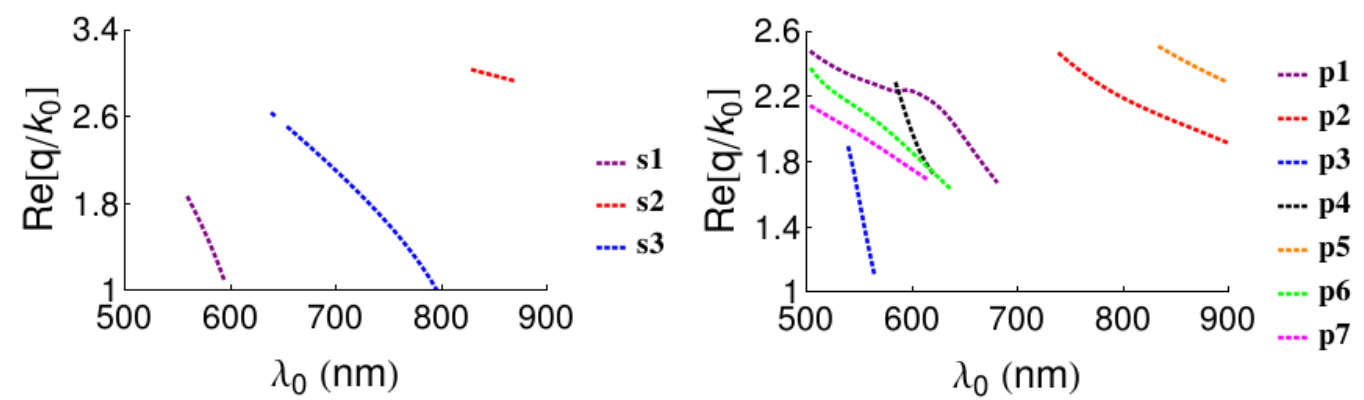

Figure 3: Real parts of the normalized wavenumbers $q / k_{0}$ of $s$ - and $p$-polarized SPP waves obtained after solving the relevant canonical boundary-value problem.
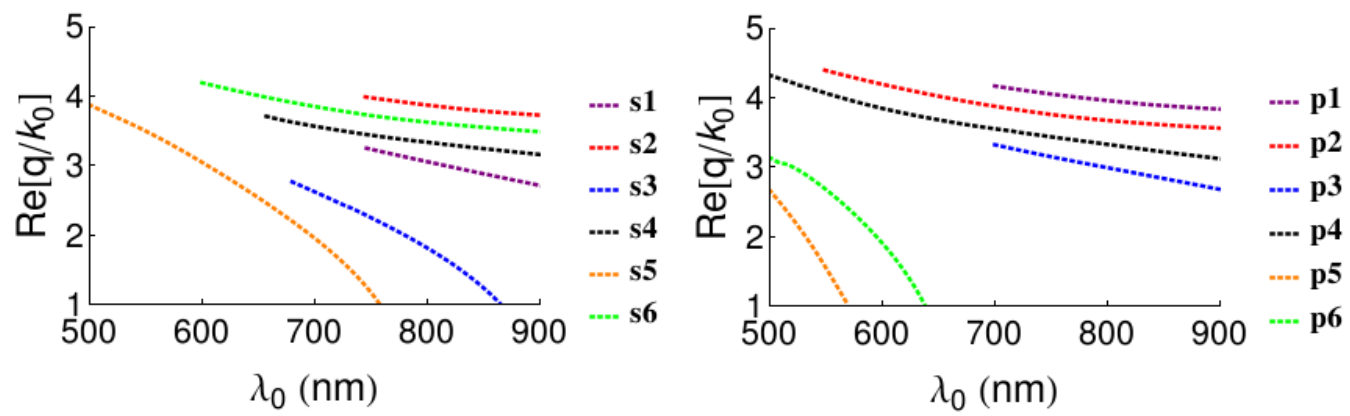

Figure 4: Real parts of the normalized wavenumbers $q / k_{0}$ of $s$ - and $p$-polarized WGMs obtained after solving the relevant canonical boundary-value problem.

\subsection{Absorptances and Correlation with Predictions}

Calculations of $A_{\mathrm{s}}$ and $A_{\mathrm{p}}$ as functions of $\lambda_{0} \in[500,900] \mathrm{nm}$ were made for the chosen triple-p-i-n-junction tandem solar cell with a 2D PCBR. In addition, we computed the spectrums of the useful absorptances

$$
\left.\begin{array}{l}
\bar{A}_{\mathrm{s}}^{\mathrm{sc}}=\left.\bar{A}^{\mathrm{sc}}\right|_{\bar{a}_{\mathrm{p}}=0} \\
\bar{A}_{\mathrm{p}}^{\mathrm{sc}}=\left.\bar{A}^{\mathrm{sc}}\right|_{\bar{a}_{\mathrm{s}}=0}
\end{array}\right\} \text {. }
$$

The spectrums of $A_{\mathrm{s}}, A_{\mathrm{p}}, \bar{A}_{\mathrm{s}}^{\text {sc }}$, and $\bar{A}_{\mathrm{p}}^{\mathrm{sc}}$ for $\lambda_{0} \in[500,900] \mathrm{nm}$ for solar cells with and without corrugations ( $L_{\mathrm{g}}=80 \mathrm{~nm}$ and $L_{\mathrm{g}}=0$, respectively) were examined for several combinations of $\theta$ and $\psi$ [39]. For the sake of illustration, data are presented in Figs. 5-8 only for the following two directions of incidence:

(1) $\left\{\psi=1^{\circ}, \theta=1^{\circ}\right\}$, and

(2) $\left\{\psi=45^{\circ}, \theta=15^{\circ}\right\}$.

The choices of $1^{\circ}$ instead of $0^{\circ}$ for the incidence angles help avoid spurious results associated with the computation of distinct eigenvalues of $\breve{\mathbf{P}}(z)$ when the RCWA is implemented. Also shown in these figures are the spectrums of the useful absorptances $\bar{A}_{\mathrm{s}}^{\mathrm{sc} m}$ and $\bar{A}_{\mathrm{p}}^{\mathrm{sc} m}$ in the $m$ th $p-i-n$ junction, $m \in\{1,2,3\}$, for incident $s$ - and $p$-polarized plane waves, respectively.

The excitation of a GWM is marked by an absorptance peak. Therefore, values of $\lambda_{0} \in[500,900] \mathrm{nm}$ for which the solutions of the two canonical problems (with the assumption that $L_{\mathrm{w}}=L_{\mathrm{a}}=0$ ) predicted the excitation of SPP waves and WGMs for $\theta \in\left[0^{\circ}, 2^{\circ}\right] \cup\left[14^{\circ}, 16^{\circ}\right]$ are also identified in Figs. 5-8. Red arrows indicate the excitation of SPP waves that matched with both total absorptances $\left(A_{\mathrm{s}}\right.$ and $\left.A_{\mathrm{p}}\right)$ and useful 
absorptances $\left(\bar{A}_{\mathrm{s}}^{\mathrm{sc}}\right.$ and $\left.\bar{A}_{\mathrm{p}}^{\mathrm{sc}}\right)$; black arrows indicate WGMs that matched with both total absorptances and useful absorptances; blue arrows indicate the excitation of SPP waves that correlated with total absorptances but not with useful absorptances; and purple arrows indicate the excitation of WGMs that correlated with total absorptances but not with useful absorptances.

\subsubsection{Case 1: $\left\{\psi=1^{\circ}, \theta=1^{\circ}\right\}$}

Spectrums of $A_{\mathrm{s}}, A_{\mathrm{p}}, \bar{A}_{\mathrm{s}}^{\mathrm{sc}}$ and $\bar{A}_{\mathrm{p}}^{\mathrm{sc}}$ for $\left\{\psi=1^{\circ}, \theta=1^{\circ}\right\}$ calculated with $L_{\mathrm{g}}=80 \mathrm{~nm}$ are presented in Fig. 5 . Also, spectrums of the same quantities calculated with $L_{\mathrm{g}}=0$ are presented in Fig. 6 for comparison. Tables II and III contain values of $\lambda_{0} \in[500,900] \mathrm{nm}$ for which the excitation of either an SPP wave or a WGM as a Floquet harmonic of order $(m, n)$ is predicted.

The $\bar{A}_{\mathrm{s}}^{\text {sc }}$-peak at $\lambda_{0} \approx 728 \mathrm{~nm}$ in Fig. 5 occurs close to the wavelength $\lambda_{0} \approx 730 \mathrm{~nm}$ predicted for the excitation of an $s$-polarized SPP wave as a Floquet harmonic of order $(1,0)$ at $\theta=0.856^{\circ}$ in Table II. This is the only $\mathrm{SPP}$ wave that correlated with peaks of both $\bar{A}_{\mathrm{s}}^{\mathrm{sc}}$ and $A_{\mathrm{s}}$.

The $A_{\mathrm{s}}$-peak in Fig. 5 at

- $\lambda_{0} \approx 845 \mathrm{~nm}$ is due to the excitation of an $s$-polarized SPP wave as a Floquet harmonic of order $(1,1)$ predicted at $\theta=0.088^{\circ}$ in Table II,

- $\lambda_{0} \approx 897 \mathrm{~nm}$ matches well with the excitation of a $p$-polarized SPP wave as a Floquet harmonic of order $(1,0)$ predicted at $\theta=0.847^{\circ}$ in Table II,

- $\lambda_{0} \approx 754 \mathrm{~nm}$ is related with the excitation of a $p$-polarized WGM as a Floquet harmonic of order $(-2,0)$ predicted at $\theta=1.145^{\circ}$ in Table III, and

- $\lambda_{0} \approx 892 \mathrm{~nm}$ is represents the excitation of a $p$-polarized WGM as a Floquet harmonic of order $(-1,1)$ predicted at $\theta=1.198^{\circ}$ in Table III,

Excitation of these GWMs correlated only with the total absorptance $A_{\mathrm{s}}$ but not with the useful absorptance $\bar{A}_{\mathrm{s}}^{\mathrm{sc}}$, which indicates that not every $\bar{A}_{\mathrm{s}}^{\mathrm{sc}}$-peak can be matched to an $A_{\mathrm{s}}$-peak that is correlated with the excitation of a GWM [21,44]. Accordingly, useful absorptance, not the overall absorptance, needs to be studied for solar cells. Contributions to the overall absorptance are made both by the semiconductor layers and the metallic PCBR, but the contribution of the latter is useless for harvesting solar energy.

The $\bar{A}_{\mathrm{p}}^{\text {sc }}$-peak in Fig. 5 at $\lambda_{0} \approx 786 \mathrm{~nm}$ is due to the excitation of an $s$-polarized WGM as a Floquet harmonic of order $(-1,0)$ predicted at $\theta=0.948^{\circ}$ in Table III. The $A_{\mathrm{p}}$-peak at

- $\lambda_{0} \approx 834 \mathrm{~nm}$ is due to the excitation of a $p$-polarized WGM as a Floquet harmonic of order $(-1,1)$ predicted at $\theta=0.838^{\circ}$ in Table III, and

- $\lambda_{0} \approx 845 \mathrm{~nm}$ matches well with the excitation of a $p$-polarized SPP wave as a Floquet harmonic of order $(1,1)$ predicted at $\theta=0.088^{\circ}$ in Table II,

On comparing Figs. 5 and 6 , we note that the GWMs are excited at $\lambda_{0}>700 \mathrm{~nm}$. Also, the total absorptance for $L_{\mathrm{g}}=80 \mathrm{~nm}$ exceeds that for $L_{\mathrm{g}}=0$ in the same spectral regime. This increase is largely due to the increases in $\bar{A}_{\mathrm{s}}^{\mathrm{sc} 3}$ and $\bar{A}_{\mathrm{p}}^{\text {sc3 }}$, i.e., in the $p-i-n$ junction closest to the PCBR. Furthermore, increases in both total and useful absorptances for $\lambda_{0} \in[634,680] \mathrm{nm}$, regardless of the polarization state of the incident light, were observed with the use of the PCBR rather than a planar backreflector. In addition, depolarization due to the two-dimensional periodicity of the PCBR is evident from the excitation of WGMs that are not of the same polarization state as the incident light.

\subsubsection{Case 2: $\left\{\psi=45^{\circ}, \theta=15^{\circ}\right\}$}

Calculated spectrums of $A_{\mathrm{s}}, A_{\mathrm{p}}, \bar{A}_{\mathrm{s}}^{\mathrm{sc}}$, and $\bar{A}_{\mathrm{p}}^{\mathrm{sc}}$ for $\left\{\psi=45^{\circ}, \theta=15^{\circ}\right\}$ with $L_{\mathrm{g}}=80 \mathrm{~nm}$ are presented in Fig 7. Also, the spectrums $A_{\mathrm{s}}, A_{\mathrm{p}}, \bar{A}_{\mathrm{s}}^{\mathrm{sc}}$, and $\bar{A}_{\mathrm{p}}^{\text {sc }}$ calculated with $L_{\mathrm{g}}=0$ for the same incident direction are 
Table II: Values of $\lambda_{0} \in[500,900] \mathrm{nm}$ (calculated at 1-nm intervals) for which the excitation of an SPP wave as a Floquet harmonic of order $(m, n)$ is predicted for $\theta \in\left[0^{\circ}, 2^{\circ}\right]$ and $\psi=1^{\circ}$, for the tandem solar cell with a 2D PCBR. The SPP waves strongly excited in Fig. 5 are highlighted in bold.

\begin{tabular}{lllll}
\hline \hline Pol. State & $\lambda_{0}(\mathrm{~nm})$ & $\operatorname{Re}\left\{q / k_{0}\right\}$ & $\theta^{\circ}$ & $(m, n)$ \\
\hline $\mathbf{s}$ & $\mathbf{7 3 0}$ & $\mathbf{1 . 8 1 6}$ & $\mathbf{0 . 8 5 6}$ & $(\mathbf{1}, \mathbf{0})$ \\
$\mathbf{s}$ & $\mathbf{8 4 5}$ & $\mathbf{2 . 9 8 8}$ & $\mathbf{0 . 0 8 8}$ & $(\mathbf{1}, \mathbf{1})$ \\
$p$ & 570 & 2.027 & 0.991 & $(1,1)$ \\
$p$ & 640 & 2.029 & 1.024 & $( \pm 1,0)$ \\
$p$ & 680 & 1.678 & 1.228 & $(1,0)$ \\
$\mathbf{p}$ & $\mathbf{8 9 7}$ & $\mathbf{2 . 9 8 8}$ & $\mathbf{0 . 8 4 7}$ & $(\mathbf{1}, \mathbf{0})$ \\
\hline \hline
\end{tabular}

Table III: Same as Table II, except that the relevant excitations of WGMs are indicated. The WGMs strongly excited in Fig. 5 are highlighted in bold.

\begin{tabular}{lllll}
\hline \hline Pol. State & $\lambda_{0}(\mathrm{~nm})$ & $\operatorname{Re}\left\{q / k_{0}\right\}$ & $\theta^{\circ}$ & $(m, n)$ \\
\hline$s$ & 663 & 3.685 & 1.334 & $(-2,1)$ \\
$s$ & 711 & 3.536 & 1.076 & $(-2,0)$ \\
$\mathbf{s}$ & $\mathbf{7 8 6}$ & $\mathbf{1 . 9 4 8}$ & $\mathbf{0 . 9 4 8}$ & $(-\mathbf{1}, \mathbf{0})$ \\
$\mathbf{s}$ & $\mathbf{8 3 4}$ & $\mathbf{2 . 9 3 8}$ & $\mathbf{0 . 8 3 8}$ & $(-\mathbf{1}, \mathbf{1})$ \\
$s$ & 898 & 3.163 & 0.902 & $(-1,1)$ \\
$\mathbf{p}$ & $\mathbf{7 5 4}$ & $\mathbf{3 . 7 5 0}$ & $\mathbf{1 . 1 4 5}$ & $(-\mathbf{2 , 0})$ \\
$p$ & 797 & 3.963 & 1.206 & $(-2,0)$ \\
$p$ & 827 & 2.910 & 1.121 & $(-1,1)$ \\
$\mathbf{p}$ & $\mathbf{8 9 2}$ & $\mathbf{3 . 1 3 8}$ & $\mathbf{1 . 1 9 8}$ & $(-\mathbf{1 , 1})$ \\
\hline \hline
\end{tabular}

presented in Fig. 8. Tables IV and V contain values of $\lambda_{0} \in[500,900] \mathrm{nm}$ for which the excitation of either an SPP wave or a WGM as a Floquet harmonic of order $(m, n)$ is predicted from analysis of Figs. 3 and 4 ..

No $\bar{A}_{\mathrm{s}}^{\text {sc }}$-peak could be correlated with the excitation of a GWM. The $A_{\mathrm{s}}$-peak at

- $\lambda_{0} \approx 800 \mathrm{~nm}$ is related to the excitation of a $p$-polarized SPP wave as a Floquet harmonic of order either $(1,0)$ or $(0,1)$ predicted at $\theta=14.952^{\circ}$ in Table IV,

- $\lambda_{0} \approx 870 \mathrm{~nm}$ is due to the excitation of a $p$-polarized SPP wave as a Floquet harmonic of order either $(-1,0)$ or $(0,-1)$ predicted at $\theta=15.880^{\circ}$ in Table IV,

- $\lambda_{0} \approx 764 \mathrm{~nm}$ is associated with the excitation of a $p$-polarized WGM as a Floquet harmonic of order $(-1,-2)$ predicted at $\theta=15.141^{\circ}$ in Table V. and

- $\lambda_{0} \approx 778 \mathrm{~nm}$ arises due to the excitation of a $p$-polarized WGM as a Floquet harmonic of order either $(-2,0)$ or $(0,-2)$ predicted at $\theta=15.411^{\circ}$ in Table $\mathrm{V}$.

The $\overline{A_{\mathrm{p}}}$-peak at $\lambda_{0} \approx 647 \mathrm{~nm}$ is related with the excitation of an $s$-polarized SPP wave as a Floquet harmonic of order $(1,1)$ at $\theta=15.529^{\circ}$ in Table IV. No other $\bar{A}_{\mathrm{p}}^{\text {sc }}$ - or $A_{\mathrm{p}}$-peak was found to be correlated with GWM excitation. These results underscore the fact that useful absorptance is not necessarily enhanced by the excitation of a GWM. However, there are useful- and total-absorptance peaks which could not predicted by the canonical boundary-value problems.

On comparing Figs. 7 and 8, increases in both total and useful absorptances for $\lambda_{0} \in[640,670] \mathrm{nm}$, regardless of the polarization state of the incident light, become evident with the use of the PCBR rather 

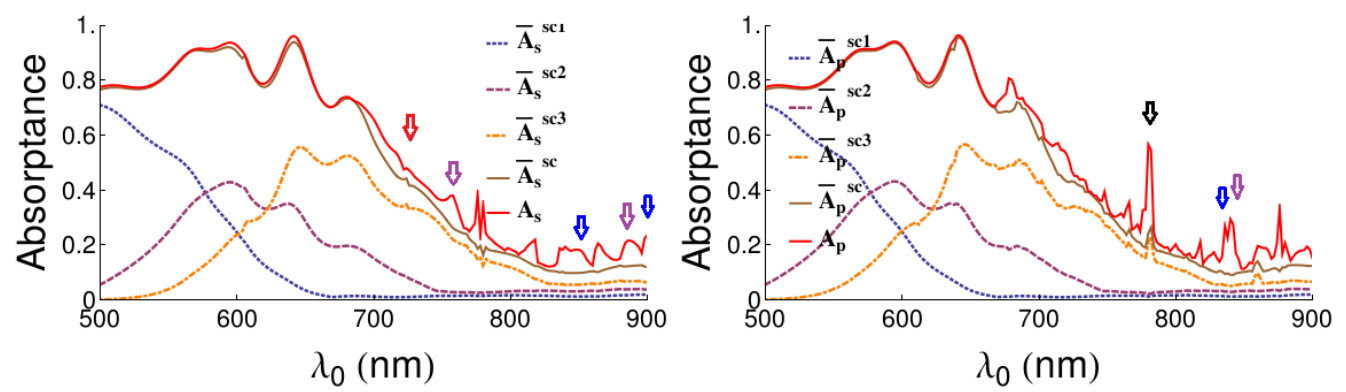

Figure 5: Spectrums of (left) $A_{\mathrm{s}}, \bar{A}_{\mathrm{s}}^{\mathrm{sc}}, \bar{A}_{\mathrm{s}}^{\mathrm{sc} 1}, \bar{A}_{\mathrm{s}}^{\mathrm{sc} 2}$, and $\bar{A}_{\mathrm{s}}^{\mathrm{sc} 3}$ and (right) $A_{\mathrm{p}}, \bar{A}_{\mathrm{p}}^{\mathrm{sc}}, \bar{A}_{\mathrm{p}}^{\mathrm{sc} 1}, \bar{A}_{\mathrm{p}}^{\mathrm{sc} 2}$, and $\bar{A}_{\mathrm{p}}^{\mathrm{sc} 3}$ of the triple- $p-i-n$-junction tandem solar cell, when $\psi=1^{\circ}$ and $\theta=1^{\circ}$. Red arrows indicate the excitation of SPP waves that matched with both total absorptances $\left(A_{\mathrm{s}}\right.$ and $\left.A_{\mathrm{p}}\right)$ and useful absorptances $\left(\bar{A}_{\mathrm{s}}^{\mathrm{sc}}\right.$ and $\left.\bar{A}_{\mathrm{p}}^{\mathrm{sc}}\right)$; black arrows indicate WGMs that matched with both total absorptances and useful absorptances; blue arrows indicate the excitation of SPP waves that correlated with total absorptances but not with useful absorptances; and purple arrows indicate the excitation of WGMs that correlated with total absorptances but not with useful absorptances.
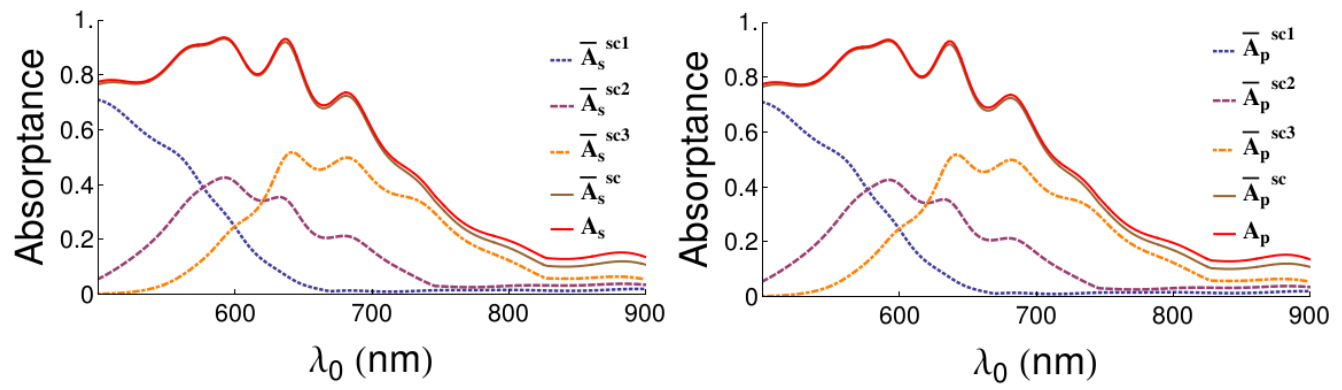

Figure 6: Same as Fig. 5 except that $L_{\mathrm{g}}=0$.

than a planar backreflector. In comparison to normal illumination (Sec. 3.2.1) for which GWMs were excited only for $\lambda_{0}>700 \mathrm{~nm}$, an SPP wave is excited at $\lambda_{0}=647 \mathrm{~nm}$ for oblique illumination. This is in accord with the blueshifts of SPP waves expected for oblique illumination $[18,42]$ as well as with the angular trends in Figs. 3 and 4.

Apart from the SPP wave excited at $\lambda_{0}=647 \mathrm{~nm}$, all other GWMs are excited at $\lambda_{0}>700 \mathrm{~nm}$. The polarization state of an excited GWM may not be the same as that of the incident light, because the 2D PCBR is a depolarizing agent. Finally, the total absorptance increases in the same spectral regime with the use of the PCBR in comparison to a planar backreflector, for either polarization state of the incident light, largely due to the increases in $\bar{A}_{\mathrm{s}}^{\mathrm{sc} 3}$ and $\bar{A}_{\mathrm{p}}^{\mathrm{sc} 3}$, i.e., in the $p-i-n$ junction closest to the PCBR.

\section{Concluding remarks}

The effect of a 2D PCBR on the absorptance of light in a triple- $p$ - $i$ - $n$-junction thin-film solar cell was studied using the RCWA. Total absorptances and useful absorptances for incident $s$ - and $p$-polarized light were computed against the free-space wavelength for two different incidence directions. Calculations were also made of the useful absorptance in each of the three $p-i-n$ junctions. Furthermore, two canonical boundaryvalue problems were solved for the prediction of GWMs. The predicted GWMs were correlated with the peaks of the total and useful absorptances for both linear polarization states.

Numerical studies led to the following conclusions: 
Table IV: Values of $\lambda_{0} \in[500,900] \mathrm{nm}$ (calculated at 1-nm intervals) for which the excitation of an SPP wave as a Floquet harmonic of order $(m, n)$ is predicted for $\theta \in\left[0^{\circ}, 2^{\circ}\right]$ and $\psi=45^{\circ}$, for the tandem solar cell backed by a 2D PCBR. The SPP waves strongly excited in Fig. 7 are highlighted in bold.

\begin{tabular}{lllll}
\hline \hline Pol. State & $\lambda_{0}(\mathrm{~nm})$ & $\operatorname{Re}\left\{q / k_{0}\right\}$ & $\theta^{\circ}$ & $(m, n)$ \\
\hline $\mathbf{s}$ & $\mathbf{6 4 7}$ & $\mathbf{2 . 5 8 1}$ & $\mathbf{1 5 . 5 2 9}$ & $(\mathbf{1 , 1})$ \\
$s$ & 690 & 2.193 & 14.231 & $(-1,-1)$ \\
$p$ & 565 & 2.272 & 15.955 & $(1,1)$ \\
$p$ & 620 & 1.734 & 14.365 & $(1,0),(0,1)$ \\
$p$ & 750 & 2.404 & 14.315 & $(-1,-1)$ \\
$\mathbf{p}$ & $\mathbf{8 0 0}$ & $\mathbf{2 . 1 9 0}$ & $\mathbf{1 4 . 9 5 2}$ & $(\mathbf{1}, \mathbf{0}),(\mathbf{0}, \mathbf{1})$ \\
$\mathbf{p}$ & $\mathbf{8 7 0}$ & $\mathbf{2 . 3 7 6}$ & $\mathbf{1 5 . 8 8 0}$ & $(-\mathbf{1}, \mathbf{0}),(\mathbf{0},-\mathbf{1})$ \\
\hline \hline
\end{tabular}

Table V: Same as Table IV, except that the relevant excitations of WGMs are indicated. The WGMs strongly excited in Fig. 7 are highlighted in bold.

\begin{tabular}{lllll}
\hline \hline Pol. State & $\lambda_{0}(\mathrm{~nm})$ & $\operatorname{Re}\left\{q / k_{0}\right\}$ & $\theta^{\circ}$ & $(m, n)$ \\
\hline$s$ & 732 & 3.484 & 15.004 & $(-2,0),(0,-2)$ \\
$s$ & 807 & 3.858 & 14.931 & $(-2,0),(0,-2)$ \\
$\mathbf{p}$ & $\mathbf{7 6 4}$ & $\mathbf{4 . 0 2 3}$ & $\mathbf{1 5 . 1 4 1}$ & $(-\mathbf{1},-\mathbf{2})$ \\
$\mathbf{p}$ & $\mathbf{7 7 8}$ & $\mathbf{3 . 7 0 6}$ & $\mathbf{1 5 . 4 1 1}$ & $(-\mathbf{2}, \mathbf{0}),(\mathbf{0},-\mathbf{2})$ \\
$p$ & 664 & 3.641 & 14.287 & $(-2,1)$ \\
$p$ & 732 & 3.477 & 15.355 & $(-2,0),(0,-2)$ \\
$p$ & 821 & 3.925 & 15.098 & $(-2,0),(0,-2)$ \\
\hline \hline
\end{tabular}

- Regardless of the illumination direction and the polarization state of the incident light, increases in useful and total absorptances for $\lambda_{0}<700 \mathrm{~nm}$ arise from the replacement of a planar backreflector by a 2D PCBR.

- The triple- $p$ - $i-n$-junction tandem solar cell made of a-Si alloys is highly absorbing for $\lambda_{0}<700 \mathrm{~nm}$, so that the excitation of SPP waves in this regime is unnoticeable.

- Both SPP waves and WGMs are excited for $\lambda_{0}>700 \mathrm{~nm}$ for both normal and oblique illumination. An SPP wave excited at $\lambda_{0}=647 \mathrm{~nm}$ for oblique illumination is in accord with blueshifting of SPP waves with increasing obliqueness of illumination.

- Some of the excited GWMs directly contribute to the increase in useful absorptance of the solar cell backed by a $2 \mathrm{D}$ PCBR. This increase is largely due to enhanced absorptance in the $p$ - $i$ - $n$ junction closest to the 2D PCBR.

- Depolarization due to the two-dimensional periodicity of the PCBR is evident from the excitation of GWMs that are not of the same polarization state as the incident light.

- Excitation of certain GWMs could be correlated with the total absorptance but not with the useful absorptance.

When devising light-trapping strategies, the useful, but not the total absorptance needs to be focused on. While reduction of reflectance is a worthwhile objective, meeting it will not necessarily boost the useful absorptance. We conclude with the recommendation that material and geometric parameters need to be optimized for efficiency enhancement. 

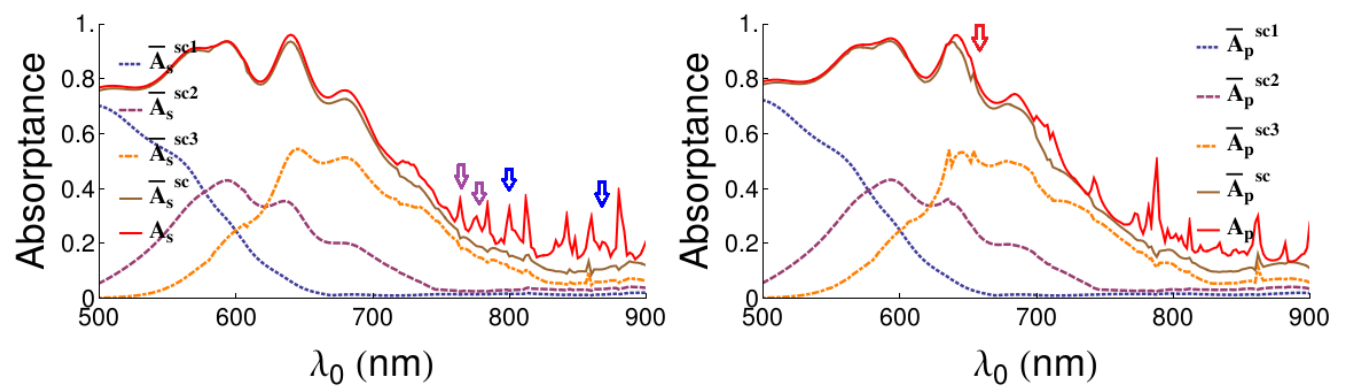

Figure 7: Same as Fig. 5, except that $\theta=15^{\circ}$ and $\psi=45^{\circ}$.
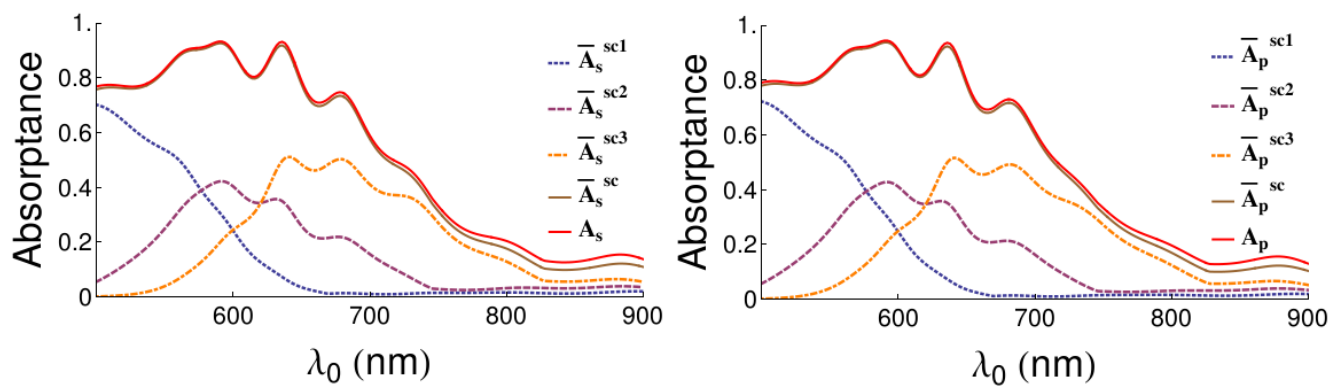

Figure 8: Same as Fig. 7, except that $L_{\mathrm{g}}=0$.

Note. This paper is substantially based on a paper titled, "On optical-absorption peaks in a nonhomogeneous dielectric material over a two-dimensional metallic surface-relief grating," presented at the SPIE Optics and Photonics conference Nanostructured Thin Films X, held August 5-11, 2017 in San Diego, California, United States.

Acknowledgments. F. Ahmad thanks the Graduate School and the College of Engineering, Pennsylvania State University, for a University Graduate Fellowship during the first year of his doctoral studies. A. Lakhtakia thanks the Charles Godfrey Binder Endowment at the Pennsylvania State University for ongoing support of his research. The research of F. Ahmed and A. Lakhtakia is partially supported by US National Science Foundation (NSF) under grant number DMS-1619901. The research of T.H. Anderson, B.J. Civiletti, and P.B. Monk is partially supported by the US National Science Foundation (NSF) under grant number DMS-1619904.

\section{References}

[1] R. Singh, "Why silicon is and will remain the dominant photovoltaic material," J. Nanophoton. 3(1), $032503(2009)$.

[2] D. E. Carlson and C. R. Wronski, "Amorphous silicon solar cell," Appl. Phys. Lett. 28(11), 671-673 (1976).

[3] M. A. Green, "Thin-film solar cells: review of materials, technologies and commercial status," J. Mater. Sci: Mater. Electron. 18(S1), 15-19 (2007).

[4] R. Singh, G. F. Alapatt, and A. Lakhtakia, "Making solar cells a reality in every home: Opportunities and challenges for photovoltaic device design," IEEE J. Electron. Dev. Soc. 1(6), 129-144 (2013). 
[5] R. J. Martín-Palma and A. Lakhtakia, "Progress on bioinspired, biomimetic, and bioreplication routes to harvest solar energy," Appl. Phys. Rev. 4(2), 021103 (2017).

[6] I. G. Kavakli and K. Kantarli, "Single and double-layer antireflection coatings on silicon," Turk. J. Phys. 26(5), 349-354 (2002).

[7] S. K. Dhungel, J. Yoo, K. Kim, S. Jung, S. Ghosh, and J. Yi, "Double-layer antireflection coating of $\mathrm{MgF}_{2} / \mathrm{SiN}_{x}$ for crystalline silicon solar cells," J. Korean Phys. Soc. 49(3), 885-889 (2006).

[8] S. A. Boden and D. M. Bagnall, "Sunrise to sunset optimization of thin film antireflective coatings for encapsulated, planar silicon solar cells," Prog. Photovoltaics Res. Appl. 17(4), 241-252 (2009).

[9] W. H. Southwell, "Pyramid-array surface-relief structures producing antireflection index matching on optical surfaces," J. Opt. Soc. Am. A 8(3), 549-553 (1991).

[10] K. C. Sahoo, M.-K. Lin, E.-Y. Chang, T. B. Tinh, Y. Li, and J.-H. Huang, "Silicon nitride nanopillars and nanocones formed by nickel nanoclusters and inductively coupled plasma etching for solar cell application," Jpn. J. Appl. Phys. 48(12), 126508 (2009).

[11] P. Sheng, A. N. Bloch, and R. S. Stepleman, "Wavelength-selective absorption enhancement in thin-film solar cells," Appl. Phys. Lett. 43(6), 579-581 (1983).

[12] C. Heine and R. H. Morf, "Submicrometer gratings for solar energy applications," Appl. Opt. 34(14), 2476-2482 (1995).

[13] M. Solano, M. Faryad, A. S. Hall, T. E. Mallouk, P. B. Monk, and A. Lakhtakia, "Optimization of the absorption efficiency of an amorphous-silicon thin-film tandem solar cell backed by a metallic surfacerelief grating," Appl. Opt. 52(5), 966-979 (2013); erratum: 54(3), 398-399 (2015).

[14] Y. Zhang, B. Jia, Z. Ouyang, and M. Gu, "Influence of rear located silver nanoparticle induced light losses on the light trapping of silicon wafer-based solar cells," J. Appl. Phys. 116(12), 124303 (2014).

[15] L. M. Anderson, "Parallel-processing with surface plasmons, A new strategy for converting the broad solar spectrum," Proc. 16th IEEE Photovoltaic Specialist Conf. 1(1), 371-377 (1982).

[16] L. M. Anderson, "Harnessing surface plasmons for solar energy conversion," Proc. SPIE 408(1), 172-178 (1983).

[17] M. G. Deceglie, V. E. Ferry, A. P. Alivisatos, and H. A. Atwater, "Design of nanostructured solar cells using coupled optical and electrical modeling," Nano Lett. 12(6), 2894-2900 (2012).

[18] M. Faryad and A. Lakhtakia, "Enhancement of light absorption efficiency of amorphous-silicon thin-film tandem solar cell due to multiple surface-plasmon-polariton waves in the near-infrared spectral regime," Opt. Eng. 52(8), 087106 (2013); errata: 53(12), 129801 (2014).

[19] M. E. Solano, G. D. Barber, A. Lakhtakia, M. Faryad, P. B. Monk, and T. E. Mallouk, "Buffer layer between a planar optical concentrator and a solar cell," AIP Advances 5(9), 097150 (2015).

[20] L. Liu, G. D. Barber, M. V. Shuba, Y. Yuwen, A. Lakhtakia, T. E. Mallouk, and T. S. Mayer, "Planar light concentration in micro-Si solar cells enabled by a metallic grating-photonic crystal architecture," ACS Photon. 3(4), 604-610 (2016).

[21] L. Liu, M. Faryad, A. S. Hall, G. D. Barber, S. Erten, T. E. Mallouk, A. Lakhtakia, and T. S. Mayer, "Experimental excitation of multiple surface-plasmon-polariton waves and waveguide modes in a onedimensional photonic crystal atop a two-dimensional metal grating," J. Nanophotonics 9(1), 093593 (2015). 
[22] F.-J. Haug, K. Söderström, A. Naqavi, and C. Ballif, "Excitation of guided-mode resonances in thin film silicon solar cells," MRS Symp. Proc. 1321(1), 123-128 (2011).

[23] T. Khaleque and R. Magnusson, "Light management through guided-mode resonances in thin-film silicon solar cells," J. Nanophotonics 8(1), 083995 (2014).

[24] J. A. Polo Jr., T. G. Mackay, and A. Lakhtakia, Electromagnetic Surface Waves: A Modern Perspective, Elsevier, Waltham, MA, USA (2013).

[25] A. W. Snyder and J. D. Love, Optical Waveguide Theory, Chapman and Hall, New York, NY, USA (1983).

[26] T. H. Anderson, M. Faryad, T. G. Mackay, A. Lakhtakia, and R. Singh, "Combined optical-electrical finite-element simulations of thin-film solar cells with homogeneous and nonhomogeneous intrinsic layers," J. Photon. Energy 6(2), 025502 (2016).

[27] T. H. Anderson, T. G. Mackay, and A. Lakhtakia, "Enhanced efficiency of Schottky-barrier solar cell with periodically nonhomogeneous indium gallium nitride layer," J. Photon. Energy 7(1), 014502 (2017).

[28] A. S. Hall, M. Faryad, G. D. Barber, L. Liu, S. Erten, T. S. Mayer, A. Lakhtakia, and T. E. Mallouk, "Broadband light absorption with multiple surface plasmon polariton waves excited at the interface of a metallic grating and photonic crystal," ACS Nano 7(6), 4995-5007 (2013).

[29] M. Faryad, A. S. Hall, G. D. Barber, T. E. Mallouk, and A. Lakhtakia, "Excitation of multiple surfaceplasmon-polariton waves guided by a periodically corrugated interface of a metal and a periodic multilayered isotropic dielectric material," J. Opt. Soc. Am. B 29(4), 704-713 (2012).

[30] M. Faryad, L. Liu, T. S. Mayer, and A. Lakhtakia, "Optical and electrical modeling of an amorphoussilicon tandem solar cell with nonhomogeneous intrinsic layers and a periodically corrugated backreflector," Proc. SPIE 8823(1), 882306 (2013).

[31] M. V. Shuba, M. Faryad, M. E. Solano, P. B. Monk, and A. Lakhtakia, "Adequacy of the rigorous coupled-wave approach for thin-film silicon solar cells with periodically corrugated metallic backreflectors: spectral analysis," J. Opt. Soc. Am. A 32(7), 1222-1230 (2015).

[32] A. S. Ferlauto, G. M. Ferreira, J. M. Pearce, C. R. Wronski, R. W. Collins, X. Deng, and G. Ganguly, "Analytical model for the optical functions of amorphous semiconductors from the near-infrared to ultraviolet: Applications in thin film photovoltaics," J. Appl. Phys. 92(5), 2424-2436 (2002).

[33] S. J. Fonash, Solar Cell Device Physics, Academic Press, Burlington, MA, USA (2010).

[34] http://refractiveindex.info/?group=METALS/material=Silver (accessed 9 July 2017).

[35] E. N. Glytsis and T. K. Gaylord, "Rigorous three-dimensional coupled-wave diffraction analysis of single and cascaded anisotropic gratings," J. Opt. Soc. Am. A 4(11), 2061-2080 (1987).

[36] M. Onishi, K. Crabtree, and R. A. Chipman, "Formulation of rigorous coupled-wave theory for gratings in bianisotropic media," J. Opt. Soc. Am. A 28(8), 1747-1758 (2011).

[37] E. Kreyszig, Advanced Engineering Mathematics, 10th edn., Wiley, Hoboken, NJ, USA (2011).

[38] H. Lütkepohl, Handbook of Matrices, Wiley, Chichester, United Kingdom (1996).

[39] F. Ahmad, T. H. Anderson, B. J. Civiletti, P. B. Monk, and A. Lakhtakia, "On optical-absorption peaks in a nonhomogeneous dielectric material over a two-dimensional metallic surface-relief grating," Proc. SPIE 10356(1), 103560I (2017). 
[40] M. F. Iskander, Electromagnetic Fields and Waves, 2nd edn., Waveland Press, Long Grove, IL, USA (2013).

[41] M. V. Shuba and A. Lakhtakia, "Splitting of absorptance peaks in absorbing multilayer backed by a periodically corrugated metallic reflector," J. Opt. Soc. Am. A 33(4), 779-784 (2016).

[42] J. Dutta, S. A. Ramakrishna, and A. Lakhtakia, "Characteristics of surface plasmon-polariton waves excited on 2D periodically patterned columnar thin films of silver," J. Opt. Soc. Am. A 33(9), 1697-1704 (2016).

[43] X.-Y. Gao, L. Liang, and Q.-G. Lin, "Analysis of the optical constants of aluminum-doped zinc-oxide films by using the single-oscillator model," J. Korean Phys. Soc. 57(4), 710-714 (2010).

[44] M. Faryad and A. Lakhtakia, "Grating-coupled excitation of multiple surface plasmon-polariton waves," Phys. Rev. A 84(3), 033852 (2011). 